\title{
Utility of invasive electroencephalography in children 3 years old and younger with refractory epilepsy
}

\author{
Rafael Uribe-Cardenas, MD, MHS, ${ }^{1}$ Andre E. Boyke, MS, ${ }^{2}$ Justin T. Schwarz, MD, ${ }^{1}$ \\ Peter F. Morgenstern, MD, ${ }^{1}$ Jeffrey P. Greenfield, MD, PhD, ${ }^{1}$ Theodore H. Schwartz, MD, ${ }^{1}$ \\ James T. Rutka, MD, PhD, ${ }^{3}$ James Drake, MBBCh, ${ }^{3}$ and Caitlin E. Hoffman, MD'
}

1Department of Neurological Surgery, NewYork-Presbyterian Hospital/Weill Cornell Medical Center, New York; ${ }^{2}$ Montefiore
Medical Center, New York, New York; and 'Department of Neurosurgery, Hospital for Sick Children, Toronto, Ontario, Canada

OBJECTIVE Early surgical intervention for pediatric refractory epilepsy is increasingly advocated as surgery has become safer and data have demonstrated improved outcomes with early seizure control. There is concern that the risks associated with staged invasive electroencephalography (EEG) in very young children outweigh the potential benefits. Here, the authors present a cohort of children with refractory epilepsy who were referred for invasive monitoring, and they evaluate the role and safety of staged invasive EEG in those 3 years old and younger.

METHODS The authors conducted a retrospective review of children 3 years and younger with epilepsy, who had been managed surgically at two institutions between 2001 and 2015. A cohort of pediatric patients older than 3 years of age was used for comparison. Demographics, seizure etiology, surgical management, surgical complications, and adverse events were recorded. Statistical analysis was completed using Stata version 13. A p $<0.05$ was considered statistically significant. Fisher's exact test was used to compare proportions.

RESULTS Ninety-four patients (45 patients aged $\leq 3$ [47.9\%]) and 208 procedures were included for analysis. Eighty-six procedures (41.3\%) were performed in children younger than 3 years versus 122 in the older cohort (58.7\%). Forty-two patients underwent grid placement (14 patients aged $\leq 3$ [33.3\%]); 3 of them developed complications associated with the implant (3/42 [7.14\%]), none of whom were among the younger cohort. Across all procedures, 11 complications occurred in the younger cohort versus 5 in the older patients (11/86 [12.8\%] vs $5 / 122[4.1 \%], p=0.032$ ). Two adverse events occurred in the younger group versus 1 in the older group (2/86 [2.32\%] vs 1/122 [0.82\%], $p=0.571)$. Following grid placement, $13 / 14$ younger patients underwent guided resections compared to $20 / 28$ older patients $(92.9 \%$ vs $71.4 \%, p=0.23)$.

CONCLUSIONS While overall complication rates were higher in the younger cohort, subdural grid placement was not associated with an increased risk of surgical complications in that population. Invasive electrocorticography informs management in very young children with refractory, localization-related epilepsy and should therefore be used when clinically indicated.

https://thejns.org/doi/abs/10.3171/2020.6.PEDS19504

KEYWORDS subdural grids; invasive electrocorticography; children; refractory epilepsy; localization; epileptogenic zone; electroencephalography

$\mathrm{E}$ PILEPSY affects $0.5 \%-1 \%$ of children. ${ }^{1}$ The condition in approximately $25 \%$ of these patients is medically refractory. ${ }^{2,3}$ In utero insults to the central nervous system and aberrant neuronal migration and development can cause epilepsy in infants and young children, which is refractory to pharmacological therapy. ${ }^{4}$ Refractory epilepsy leads to cognitive and functional decline. ${ }^{5}$ Although greater than $50 \%$ of children with epilepsy become seizure free in adulthood, there is a relative paucity of knowledge about the overall outcomes of prolonged epilepsy in very young children. ${ }^{6}$ Early intervention is thought to improve outcomes by limiting the effects of refractory seizures on development and by taking advantage of greater plasticity at younger ages, resulting in better recovery from surgical morbidity. ${ }^{7,8}$ It also allows for decreased exposure to antiepileptic medications, which can have deleterious effects on cognitive development. ${ }^{9,10}$ Contrary to historical paradigms, early surgical intervention is increasingly advocated for these patients. ${ }^{11}$

The success of resective epilepsy surgery relies on pre- 
operative identification of the epileptogenic zone. ${ }^{12,13}$ Noninvasive localization utilizing techniques such as video electroencephalography (EEG), functional neuroimaging, PET, SPECT, and magnetoencephalography has shown favorable results. ${ }^{14,15}$ However, these techniques are not always sufficient to identify the surgical target. Discordant data from these modalities or evidence of the epileptogenic zone residing in eloquent cortex requires invasive intracranial monitoring to guide resection. ${ }^{16,17}$ Additionally, the younger pediatric population is challenging to evaluate noninvasively given the varying patterns of seizure semiology, rapid generalization, and the varying radiographic changes observed with evolving patterns of myelination. ${ }^{18}$ While subdural grid implantation is a commonly employed method for this purpose in adults and older children, there is less robust evidence of its safety and efficacy in younger patients.

The present study was designed to analyze the safety and efficacy of subdural grid implantation in children 3 years of age or younger as compared to a cohort of older children treated for refractory epilepsy at two tertiary care centers in North America. This work is significant for the treatment of very young pediatric patients with refractory epilepsy, as it will help guide decision-making and preoperative planning.

\section{Methods}

We conducted a retrospective review of children (age $<18$ years) with refractory epilepsy who had been managed surgically at two tertiary care institutions in North America between 2001 and 2015. Patients were dichotomized into groups based on age ( $\leq 3$ and $>3$ years old). The study was approved by the institutional review board and ethical review board at the participating institutions. Patients with a history of surgical procedures performed at other institutions were not excluded. Cases were reviewed for demographics, diagnosis, workup, operative management, complications, and clinical outcome. All surgical procedures performed were reviewed, and only procedures related to epilepsy management were included for analysis. Patients with hemispheric malformations who were not candidates for focal resections were excluded from analysis. Adverse events were defined as any unfavorable clinical event encountered during the postoperative period or clinical follow-up that was not directly caused by the surgical procedure. Surgical complications were defined as any negative clinical event that resulted as a direct consequence of surgical intervention.

Data analysis was completed using Stata version 13 (StataCorp LP). A t-test was used for comparisons of means, and Fisher's exact test was used to compare proportions. A p $<0.05$ was used for statistical significance.

\section{Results}

A total of 94 patients were included for analysis (45 patients with an age $\leq 3$ years [47.9\%]; Table 1). Forty-two patients underwent grid placement (14 patients aged $\leq 3$ years [33.3\%]). The mean number of surgical procedures performed per patient was 2.2 (range 1-10), for a total of 208 surgical interventions ( 86 procedures for patients $\leq 3$
TABLE 1. Demographic and surgical data

\begin{tabular}{|c|c|c|c|}
\hline Factor & $\begin{array}{c}\leq 3 \text { Years } \\
\text { Old }\end{array}$ & $\begin{array}{c}>3 \text { Years } \\
\text { Old }\end{array}$ & $\mathrm{p}$ Value \\
\hline No. of patients (\%) & $45(47.9)$ & $49(52.1)$ & - \\
\hline No. of surgical procedures (\%) & $86(41.3)$ & $122(58.7)$ & - \\
\hline $\begin{array}{l}\text { Sex, no. }(\%) \\
\quad M \\
\text { F }\end{array}$ & $\begin{array}{l}20(44.4) \\
25(55.6)\end{array}$ & $\begin{array}{l}27(55.1) \\
22(44.9)\end{array}$ & 0.409 \\
\hline Total no. of grid cases (\%) & $14(33.3)$ & $28(66.7)$ & 0.041 \\
\hline $\begin{array}{l}\text { Resections after grid place- } \\
\text { ment, no./total (\%) }\end{array}$ & 13/14 (92.9) & $20 / 28(71.4)$ & 0.23 \\
\hline
\end{tabular}

Boldface type indicates statistical significance.

years old, 122 procedures for patients $>3$ years old; Table 2). Eight of the 42 patients (19\%) who underwent grid placement had a history of a previous craniotomy. Eight of the 42 patients $(19 \%)$ had a grid placed that was subsequently removed without undergoing subsequent resection. Only one of those 8 patients was younger than 3 years old.

Across all procedures, there were 3 adverse events (3/208 [1.44\%]; Table 3). Two adverse events occurred in the younger cohort versus 1 adverse event in the older cohort $(2 / 86[2.32 \%]$ vs $1 / 122$ [0.82\%], $\mathrm{p}=0.571)$. The adverse event in the older cohort consisted of a motor deficit after resection of a recurrent temporal lobe tumor. This deficit was a foot drop after prolonged bed rest and was not directly related to resection; therefore, it was not included as a surgical complication. Adverse events in the younger age group included a delayed wound dehiscence over a bone plate and an iliac vein thrombosis.

Across all procedures, there were 16 surgical complications (16/208 [7.7\%]). Eleven complications occurred in the group $\leq 3$ years versus 5 in the older patients $(11 / 86$ [12.8\%] vs 5/122 [4.1\%], $p=0.032$; Table 3 ). The most common surgical complications in the younger cohort were vascular injuries (6/86 [7.0\%]), followed by infection $(3 / 86$ [3.5\%]). Vascular complications in this group included an epidural hematoma after focal resection, a subdural hematoma after hemispherectomy, vascular injury during resection of cortical dysplasia, stroke after hemispherectomy, vascular injury during focal temporal resection, and vascular injury during hemispherectomy. Infections included meningitis after hemispherectomy in 2 cases and meningitis after focal resection in a third patient. Only 1 vascular complication occurred in the older cohort (subdural hematoma after grid placement). There were 3 infections in that group: empyema and abscess after grid/depth electrode implantation, meningitis after focal resection, and a wound infection after grid placement (Table 4).

With respect to grid-related procedures and complications, 42 patients underwent grid placement (14 patients aged $\leq 3$ years [33.3\%]). Twenty-three of these 42 patients underwent surgery at one institution and 19 at the other. The difference in the number of complications in all patients who had undergone grid placement between the two hospitals was not statistically significant $(2 / 23$ [8.7\%] vs $3 / 19$ [15.8\%], $\mathrm{p}=0.644$ ). No patients in the younger group developed grid-related complications, as compared to 3 
TABLE 2. Breakdown of surgical procedures

\begin{tabular}{lrr}
\hline \multicolumn{1}{c}{ Procedure } & No. & $\%$ \\
\hline Grid placement & 13 & 6.3 \\
\hline Grid \& depth placement & 29 & 13.9 \\
\hline Depth alone placement & 1 & 0.5 \\
\hline Grid removal & 8 & 3.8 \\
\hline ECoG & 2 & 1.0 \\
\hline ECoG \& resection & 1 & 0.5 \\
\hline Focal resection & 64 & 30.8 \\
\hline Repeat focal resection & 23 & 11.1 \\
\hline VNS & 6 & 2.9 \\
\hline VNS battery & 3 & 1.4 \\
\hline VNS removal & 1 & 0.5 \\
\hline Corpus callosotomy & 7 & 3.4 \\
\hline Hemispherotomy & 28 & 13.5 \\
\hline Repeat hemispherotomy & 11 & 5.3 \\
\hline RNS & 4 & 1.9 \\
\hline RNS battery & 2 & 1.0 \\
\hline RNS removal & 0 & 0.0 \\
\hline Epidural & 1 & 0.5 \\
\hline Subdural & 1 & 0.5 \\
\hline Hygroma & 1 & 0.5 \\
\hline Empyema & 1 & 0.5 \\
\hline Shunt & 1 & 0.5 \\
\hline Total & & 100.0 \\
\hline
\end{tabular}

ECoG = electrocorticography; RNS = responsive neurostimulator; VNS = vagus nerve stimulator.

patients in the older group $(0 / 14$ [0\%] vs $3 / 28$ [10.7\%], $\mathrm{p}=$ $0.539)$. Adverse events in grid patients were $1 / 14(7.14 \%)$ in those aged $\leq 3$ years and $1 / 28(3.6 \%)$ in those aged $>3$ years $(p>0.999)$. Six patients from the older cohort had a history of prior craniotomy $(6 / 28$ [21.4\%]) compared to 2 patients in the younger cohort (2/14 [14.3\%], $\mathrm{p}=0.697)$. Of the 3 patients in the older cohort who developed gridrelated complications, 2 had a history of prior craniotomies. None of the patients in the younger cohort with prior craniotomy experienced a grid-related complication.

Following grid placement, 13 patients with an age $\leq 3$ years underwent guided resections compared to 20 patients with an age $>3$ years (13/14 [92.9\%] vs 20/28 [71.4\%], p $=0.23$; Table 1). Of the 13 cases in the younger cohort that underwent resections after grid placement, 12 (92.3\%) had guided resections ( 7 had single lesionectomies and 5 had multilobar resections) and 1 (7.7\%) underwent hemispherotomy. Three of these patients from the younger cohort (3/13 [23.1\%]) who underwent initial focal resections eventually required a hemispherotomy ( 2 underwent lesionectomy and 1 underwent multilobar resection).

All of the patients in the older cohort who had surgery after grid placement had focal resections after implantation (18/20 [90\%] lesionectomies and 2/20 [10\%] multilobar resections). Five patients required subsequent surgeries for persistent seizures, two of which were hemispheric disconnections $(2 / 20[10 \%])$. The total proportion
TABLE 3. Complications and adverse events by group

\begin{tabular}{lcll}
\hline Event & $\leq 3$ Years Old & $>3$ Years Old & p Value \\
\hline All procedures & & & \\
\hline Surgical complications & $11 / 86(12.8)$ & $5 / 122(4.1)$ & 0.032 \\
\hline Adverse events & $2 / 86(2.33)$ & $1 / 122(0.82)$ & 0.571 \\
\hline Grid procedures & & & \\
\hline Surgical complications & $0 / 14(0.0)$ & $3 / 28(10.7)$ & 0.539 \\
\hline Adverse events & $1 / 14(7.14)$ & $1 / 28(3.6)$ & $>0.999$ \\
\hline
\end{tabular}

Values are expressed as number/total (\%), unless indicated otherwise. Boldface type indicates statistical significance.

of patients in both cohorts who eventually underwent a hemispheric disconnection was 4/13 (30.8\%) in the group $\leq 3$ years and 2/20 $(10.0 \%)$ in the older cohort $(\mathrm{p}=0.182)$.

\section{Discussion}

Very young children with medically refractory epilepsy are a challenging population to manage. Staged surgery can be fraught with complications because of a decreased threshold for blood loss, the presence of thinner subcutaneous tissues, which can easily erode with implanted devices, and the inherent challenges and risks of keeping a child in bed during monitoring. We sought to investigate whether subdural grid monitoring in children aged 3 years or younger carries a higher risk and if the resultant risks are outweighed by the potential benefit with respect to informing second-stage surgery. Our results show that this group does not have an increased risk of overall adverse events compared to older children. Additionally, while the overall rate of surgical complications in younger children was higher, there were no grid-related complications in this younger group. While this finding could be confounded by a higher rate of prior craniotomy in the older children, there was no similar increase in complications in the younger children who had undergone prior craniotomy.

Vascular injuries and infections were the most common surgical complications in children under 3 years. This finding is not surprising given the aforementioned risks of operating on very young patients with low circulating blood volumes and thin subcutaneous tissues. Nevertheless, none of these complications resulted from the use of subdural grids. These findings suggest that resective sur-

TABLE 4. Complications across all surgical procedures

\begin{tabular}{lcc}
\hline \multirow{2}{*}{ Complication } & \multicolumn{2}{c}{ No. (\%) } \\
\cline { 2 - 3 } & $\leq 3$ Years Old & $>3$ Years Old \\
\hline Infection & $3(3.5)$ & $3(2.5)$ \\
\hline Vasculart & $6(7.0)$ & $1(0.8)$ \\
\hline CSF leakage & $2(2.3)$ & $0(0)$ \\
\hline Wound healing & $0(0)$ & $1(0.8)$ \\
\hline
\end{tabular}

* Three grid-related complications occurred in the older cohort: a subdural hematoma, meningitis after grid implantation, and empyema/cerebral abscess. No grid-related complications happened in the younger cohort.

† Vascular injuries include any hemorrhagic complication (i.e., epidural, subdural, intraparenchymal hematoma, vessel injury, or thrombosis). 
gery in this age group carries a higher overall risk, but the use of subdural grids does not appear to be the causative factor behind the potentially increased morbidity in this age group.

When analyzing complications related to any procedure, it is important to keep in mind the surgical indication as well as the risk/benefit ratio associated with such intervention. We found that a higher proportion of patients 3 years of age and under who had undergone grid placement had a subsequent resection compared to the older group. This finding is relevant because it highlights the fact that the actionable information derived from grid placement justifies the risk of undergoing a staged procedure. The reported rates of resection after invasive monitoring in children vary in the literature. For example, Van Gompel et al. reported a 69\% rate of resection in their series of 198 patients, while Wellmer et al. reported a resection rate of $81.4 \%{ }^{19,20}$ Yang and colleagues reported a higher rate in a sample of pediatric patients, virtually all of whom underwent resective procedures after implantation except 4 cases ( 3 corpus callosotomies and 1 explantation without resection; 133/137 [97\%]). ${ }^{21}$ Adelson et al. reported that 20 of 31 patients $(64.5 \%)$ underwent resection after grid implantation. ${ }^{22}$ The proportion of patients who underwent resections after grid placement in our study falls within the previously reported rates and indicates that grid placement is a high-yield procedure in the management of young children with refractory epilepsy.

Additionally, our results suggest that children under 3 years of age are more likely to undergo hemispheric disconnection than older patients, although our results did not reach statistical significance. This finding is relevant because it highlights the fact that patient selection in the younger population is critical before planning invasive monitoring because of the potentially higher risk of surgical complications.

Other groups have studied the risks associated with invasive monitoring in children. ${ }^{21-26}$ However, there is a dearth of studies addressing this question in the very young pediatric population. This is perhaps attributable to the higher prevalence of hemispheric syndromes that do not require monitoring. For example, Bittar et al. reported their experience with surgery for refractory epilepsy in infants. They presented 11 cases, 7 of which had hemispheric involvement. ${ }^{7}$ Similarly, a study performed by Gowda et al. described 15 cases of catastrophic epilepsy in patients who were 6 months of age or younger. Nine patients had a hemispheric malformation, and 11 patients ultimately underwent hemispheric disconnections. None of these patients underwent subdural EEG monitoring. ${ }^{27}$

Duchowny et al. published their experience with 31 pediatric cases that underwent surgery in the first 3 years of life. ${ }^{8}$ Fourteen patients underwent hemispherotomy as the first line of treatment, followed by multilobar resections in three. Four patients required hemispherotomy or a multilobar resection after failed initial operations. Of the original cohort of 31 patients, 11 (35.5\%) underwent subdural grid placement, but it is unclear how many of those patients had tailored resections after monitoring.

Taussig et al. published a similar study focusing exclusively on invasive EEG monitoring in very young pediatric patients. ${ }^{28}$ Their cohort included 26 patients under the age of 3 years, 20 of whom were monitored using a combination of subdural grids and depth electrodes and 6 of whom were monitored using stereo-EEG (SEEG). There was no comparative cohort of either noninfants or patients not undergoing grid implantation. Interestingly, all of the patients who were monitored underwent subsequent resective surgeries: 21 had cortical resections, 1 patient had a disconnection, 2 had a combination of resection and disconnection, and 1 had a cortical resection with multiple subpial transections of functional cortex. Not all of the patients who were monitored in our series underwent further resective surgery; however, the proportion of patients who did undergo guided resections was higher in the younger cohort than in the older patient group. Given the heterogeneity of patients and pathologies, this finding could be the result of selection bias and would have to be corroborated with a larger cohort and better delineation of underlying etiology.

Taussig and colleagues' report describes their complications: 3 patients $(11.5 \%)$ had postoperative motor deficits, which they attributed to ischemic insults; 2 patients $(7.69 \%)$ developed subdural hygromas that required shunting; and 1 patient (3.84\%) developed a subdural hematoma that required evacuation. ${ }^{28}$ There were no infectious complications. Totaling the events reported by Taussig et al., we found the proportion of surgical complications to be $23.1 \%(6 / 26)$.

The proportion of patients who developed surgical complications in our cohort was comparatively lower across all patients, as well as across the implant patients (16/94 [17\%] and 5/42 [11.9\%], respectively). Johnston et al. reported a $13.1 \%$ rate of complications in their patient cohort with subdural implants (16 complications among 122 procedures), ${ }^{23}$ while Adelson et al. reported 5 complications in a cohort of 31 patients $(16.1 \%) .^{22}$ It is worth noting that neither of those reports focused exclusively on children under the age of 3 years or provided comparative analysis with nonimplanted cases. It is hard to compare our results with those of other groups, as most studies have analyzed the results of very young patients along with those of older children as a single population and because complications have not been reported consistently across the literature; some authors quantify complications using the total number of patients as the denominator, while others use the total number of procedures. Therefore, in our study, we report complications as a proportion of the number of procedures in each described cohort (Table 3).

Our study has limitations that merit discussion. The study was designed to group data from two well-established epilepsy surgery programs; however, one hospital provided a larger number of cases, which limits the ability to adjust our results by referral center. Our study will inherently be subject to selection bias by analyzing patients from institutions that have more experience handling difficult epilepsy cases. Therefore, these results would have to be corroborated in a larger study in order to be generalizable.

Many studies group patients with different seizure etiologies into a single group. It is reasonable to believe that different pathologies will have different levels of associ- 
ated surgical morbidity. In order to accurately assess the risks pertaining to different interventions, cohorts should be stratified by etiology or by form of surgical intervention. However, this would entail a larger multiinstitutional effort to accrue a larger number of patients.

Rare diseases and outcomes are easier to ascertain through a retrospective analysis. Nevertheless, there are always data that will be lost through chart review. Furthermore, the long period of time that has to be explored in order to accrue enough cases can potentially affect the interpretation of results given the influence of secular trends.

Because of the retrospective nature of our study and the rare occurrence of refractory epilepsy in very young patients, we were unable to match our sample cohorts.

It is worth noting that in recent years, SEEG has gained popularity in the US as the technique of choice for invasive intracranial monitoring in specific cases. ${ }^{29}$ This approach provides the ability to sample deeper and sometimes bilateral structures, sparing the need for a craniotomy, allowing for investigation of complex hypotheses, and offering the possibility of performing laser ablation through an indicated trajectory in certain cases. There is a paucity of literature on the use of SEEG in children, although the number of published papers is expanding as more groups adopt the technique. Taussig et al. published a review of invasive monitoring in children, comparing SEEG with subdural grid monitoring. ${ }^{30}$ Their article discusses the relative merits of both techniques while acknowledging that there are no standardized studies comparing the effectiveness of the two approaches. As with all investigative and therapeutic techniques in epilepsy, the right approach is patient specific, and all methods remain part of the armamentarium, utilized as indicated by a patient's age, comorbidities, and noninvasive data. Specifically, in the very young, SEEG presents the difficulty of maintaining accurate lead placement based on skull thickness and the inability to securely ground the required bolts. As the associated technical challenges are addressed and mitigated, this technique will certainly continue to gain significance in the assessment and treatment of refractory epilepsy in pediatric patients. As the indications for SEEG and subdural grid monitoring remain distinct, continued assessment of these complementary techniques is essential.

\section{Conclusions}

We conclude that subdural EEG monitoring impacts surgical planning and resection in appropriately selected young children with refractory epilepsy. Grid implantation did not increase surgical morbidity in our cohort. However, the benefits of invasive monitoring must be weighed against the potential for increased overall complication rates from staged surgical procedures in very young pediatric patients.

\section{References}

1. Hauser WA, Kurland LT. The epidemiology of epilepsy in Rochester, Minnesota, 1935 through 1967. Epilepsia. 1975; 16(1):1-66.
2. Walker MC, Sander JW. Difficulties in extrapolating from clinical trial data to clinical practice: the case of antiepileptic drugs. Neurology. 1997;49(2):333-337.

3. Keene DL, Loy-English I, Ventureyra EC. Long-term socioeconomic outcome following surgical intervention in the treatment of refractory epilepsy in childhood and adolescence. Childs Nerv Syst. 1998;14(8):362-365.

4. Kumar RM, Koh S, Knupp K, et al. Surgery for infants with catastrophic epilepsy: an analysis of complications and efficacy. Childs Nerv Syst. 2015;31(9):1479-1491.

5. Loddenkemper T, Holland KD, Stanford LD, et al. Developmental outcome after epilepsy surgery in infancy. Pediatrics. 2007;119(5):930-935.

6. Wirrell E, Wong-Kisiel L, Mandrekar J, Nickels K. Predictors and course of medically intractable epilepsy in young children presenting before 36 months of age: a retrospective, population-based study. Epilepsia. 2012;53(9):1563-1569.

7. Bittar RG, Rosenfeld JV, Klug GL, et al. Resective surgery in infants and young children with intractable epilepsy. J Clin Neurosci. 2002;9(2):142-146.

8. Duchowny M, Jayakar P, Resnick T, et al. Epilepsy surgery in the first three years of life. Epilepsia. 1998;39(7):737-743.

9. Bromley RL, Leeman BA, Baker GA, Meador KJ. Cognitive and neurodevelopmental effects of antiepileptic drugs. Epilepsy Behav. 2011;22(1):9-16.

10. Holmes GL. The 2008 Judith Hoyer lecture: epilepsy in children: listening to mothers. Epilepsy Behav. 2009;16(2): 193-202.

11. Wiebe S. Early epilepsy surgery. Curr Neurol Neurosci Rep. 2004;4(4):315-320.

12. Schmidt D, Stavem K. Long-term seizure outcome of surgery versus no surgery for drug-resistant partial epilepsy: a review of controlled studies. Epilepsia. 2009;50(6):1301-1309.

13. Téllez-Zenteno JF, Hernández Ronquillo L, Moien-Afshari F, Wiebe S. Surgical outcomes in lesional and non-lesional epilepsy: a systematic review and meta-analysis. Epilepsy Res. 2010;89(2-3):310-318.

14. Kilpatrick C, Cook M, Kaye A, et al. Non-invasive investigations successfully select patients for temporal lobe surgery. $J$ Neurol Neurosurg Psychiatry. 1997;63(3):327-333.

15. Thadani VM, Williamson PD, Berger R, et al. Successful epilepsy surgery without intracranial EEG recording: criteria for patient selection. Epilepsia. 1995;36(1):7-15.

16. Minassian BA, Otsubo H, Weiss S, et al. Magnetoencephalographic localization in pediatric epilepsy surgery: comparison with invasive intracranial electroencephalography. Ann Neurol. 1999;46(4):627-633.

17. Nair DR, Burgess R, McIntyre CC, Lüders H. Chronic subdural electrodes in the management of epilepsy. Clin Neurophysiol. 2008;119(1):11-28.

18. Nickels KC, Wong-Kisiel LC, Moseley BD, Wirrell EC. Temporal lobe epilepsy in children. Epilepsy Res Treat. 2012; 2012:849540.

19. Van Gompel JJ, Worrell GA, Bell ML, et al. Intracranial electroencephalography with subdural grid electrodes: techniques, complications, and outcomes. Neurosurgery. 2008; 63(3):498-506.

20. Wellmer J, von der Groeben F, Klarmann U, et al. Risks and benefits of invasive epilepsy surgery workup with implanted subdural and depth electrodes. Epilepsia. 2012;53(8):13221332.

21. Yang PF, Zhang HJ, Pei JS, et al. Intracranial electroencephalography with subdural and/or depth electrodes in children with epilepsy: techniques, complications, and outcomes. Epilepsy Res. 2014;108(9):1662-1670.

22. Adelson PD, Black PM, Madsen JR, et al. Use of subdural grids and strip electrodes to identify a seizure focus in children. Pediatr Neurosurg. 1995;22(4):174-180.

23. Johnston JM Jr, Mangano FT, Ojemann JG, et al. Complica- 
tions of invasive subdural electrode monitoring at St. Louis Children's Hospital, 1994-2005. J Neurosurg. 2006;105(5) (suppl):343-347.

24. Onal C, Otsubo H, Araki T, et al. Complications of invasive subdural grid monitoring in children with epilepsy. J Neurosurg. 2003;98(5):1017-1026.

25. Musleh W, Yassari R, Hecox K, et al. Low incidence of subdural grid-related complications in prolonged pediatric EEG monitoring. Pediatr Neurosurg. 2006;42(5):284-287.

26. Jayakar P, Duchowny M, Resnick TJ. Subdural monitoring in the evaluation of children for epilepsy surgery. J Child Neurol. 1994;9(suppl 2):61-66.

27. Gowda S, Salazar F, Bingaman WE, et al. Surgery for catastrophic epilepsy in infants 6 months of age and younger. $J$ Neurosurg Pediatr. 2010;5(6):603-607.

28. Taussig D, Dorfmüller G, Fohlen M, et al. Invasive explorations in children younger than 3 years. Seizure. 2012;21(8): 631-638.

29. Abou-Al-Shaar H, Brock AA, Kundu B, et al. Increased nationwide use of stereoencephalography for intracranial epilepsy electroencephalography recordings. J Clin Neurosci. 2018;53:132-134.

30. Taussig D, Chipaux M, Fohlen M, et al. Invasive evaluation in children (SEEG vs subdural grids). Seizure. 2020;77:43-51.

\section{Disclosures}

The authors report no conflict of interest concerning the materials or methods used in this study or the findings specified in this paper.

\section{Author Contributions}

Conception and design: Hoffman, Greenfield, Schwartz.

Acquisition of data: Uribe-Cardenas, Boyke, Morgenstern, Drake. Analysis and interpretation of data: all authors. Drafting the article: Uribe-Cardenas, Boyke. Critically revising the article: Hoffman, Uribe-Cardenas, Morgenstern, Schwartz, Rutka, Drake. Reviewed submitted version of manuscript: Hoffman, UribeCardenas, Boyke, Schwarz, Rutka. Approved the final version of the manuscript on behalf of all authors: Hoffman. Statistical analysis: Uribe-Cardenas, Boyke. Study supervision: Hoffman, Uribe-Cardenas, Greenfield, Schwartz, Drake.

\section{Correspondence}

Caitlin E. Hoffman: Weill Cornell Medical Center, NewYorkPresbyterian Hospital, New York, NY. ceh2003@med.cornell.edu. 